\title{
Field dependence of nonreciprocal magnons in chiral MnSi
}

\author{
T. Weber, ${ }^{1,2, *}$ J. Waizner, ${ }^{3}$ G. S. Tucker, ${ }^{4,5}$ R. Georgii,,${ }^{1,2}$ M. Kugler, ${ }^{1,2}$ A. Bauer, ${ }^{1}$ C. Pfleiderer, ${ }^{1}$ M. Garst, ${ }^{6}$ and P. Böni ${ }^{1}$ \\ ${ }^{1}$ Physik-Department, Technische Universität München, James-Franck-Strasse 1, 85748 Garching, Germany \\ ${ }^{2}$ Heinz, Maier-Leibnitz Zentrum, Technische Universität München, Lichtenbergstrasse 1, 85747 Garching, Germany \\ ${ }^{3}$ Institut für Theoretische Physik, Universität zu Köln, Zülpicher Strasse 77a, 50937 Köln, Germany \\ ${ }^{4}$ Laboratory for Neutron Scattering and Imaging, Paul Scherrer Institut, CH-5232 Villigen, Switzerland \\ ${ }^{5}$ Laboratory for Quantum Magnetism, École Polytechnique Fédérale de Lausanne, CH-1015 Lausanne, Switzerland \\ ${ }^{6}$ Institut für Theoretische Physik, Technische Universität Dresden, 01062 Dresden, Germany
}

(Received 18 August 2017; revised manuscript received 28 March 2018; published 5 June 2018)

\begin{abstract}
Spin waves in chiral magnetic materials are strongly influenced by the Dzyaloshinskii-Moriya interaction, resulting in intriguing phenomena like nonreciprocal magnon propagation and magnetochiral dichroism. Here, we study the nonreciprocal magnon spectrum of the archetypical chiral magnet MnSi and its evolution as a function of magnetic field covering the field-polarized and conical helix phase. Using inelastic neutron scattering, the magnon energies and their spectral weights are determined quantitatively after deconvolution with the instrumental resolution. In the field-polarized phase the imaginary part of the dynamical susceptibility $\chi^{\prime \prime}(\varepsilon, \mathbf{q})$ is shown to be asymmetric with respect to wave vectors $\mathbf{q}$ longitudinal to the applied magnetic field $\mathbf{H}$, which is a hallmark of chiral magnetism. In the helimagnetic phase, $\chi^{\prime \prime}(\varepsilon, \mathbf{q})$ becomes increasingly symmetric with decreasing $\mathbf{H}$ due to the formation of helimagnon bands and the activation of additional spin-flip and non-spin-flip scattering channels. The neutron spectra are in excellent quantitative agreement with the low-energy theory of cubic chiral magnets with a single fitting parameter being the damping rate of spin waves.
\end{abstract}

DOI: 10.1103/PhysRevB.97.224403

\section{INTRODUCTION}

The dispersion of spin waves in conventional magnetic materials with an inversion center is symmetric with respect to the wave vector $\mathbf{q}, \varepsilon(\mathbf{q})=\varepsilon(-\mathbf{q})$. Such an inversion center is, however, absent in chiral magnets, which implies, in general, an asymmetric spin-wave dispersion $\varepsilon(\mathbf{q}) \neq \varepsilon(-\mathbf{q})[1,2]$. As a consequence, magnons with wave vectors $\mathbf{q}$ and $-\mathbf{q}$ possess different group velocities, giving rise to nonreciprocal magnon propagation, which has been experimentally demonstrated, e.g., using spin-wave spectroscopy on $\mathrm{LiFe}_{5} \mathrm{O}_{8}$ [3], $\mathrm{Cu}_{2} \mathrm{OSeO}_{3}$ [4], FeGe, and Co-Zn-Mn alloys [5]. Similarly, in an inelastic scattering experiment where a certain wave vector $\mathbf{q}$ is transferred, magnons might be emitted with energy $\varepsilon(\mathbf{q})$ but cannot be absorbed at the same energy, which has been observed in the chiral magnet $\mathrm{MnSi}[6,7]$, as well as in the chiral antiferromagnet $\alpha-\mathrm{Cu}_{2} \mathrm{~V}_{2} \mathrm{O}_{7}$ [8]. In the presence of a magnetoelectric coupling, the damping of electromagnetic waves by nonreciprocal magnons also leads to magnetochiral dichroism, for example, in $\mathrm{Cu}_{2} \mathrm{OSeO}_{3}$ for microwave frequencies [9-12]. Here, we demonstrate the field-dependent development of nonreciprocal spin waves by combining high-resolution neutron spectroscopy measurements with a well-established theoretical framework.

A material class of particular interest comprises the cubic chiral magnets with crystal symmetry $P 2{ }_{1} 3$, including $\mathrm{MnSi}$, $\mathrm{Cu}_{2} \mathrm{OSeO}_{3}, \mathrm{FeGe}$, and $\mathrm{Fe}_{1-x} \mathrm{Co}_{x} \mathrm{Si}$. The advantage of this class of systems for investigating the spin dynamics is the relatively

*Corresponding author: tobias.weber@tum.de high symmetry of the cubic crystalline environment that considerably restricts the form of the low-energy theory in the limit of weak spin-orbit coupling. As a result, practically parameter free predictions for the magnon spectrum are available [13]. The Dzyaloshinskii-Moriya interaction (DMI) in these systems not only gives rise to a nonreciprocal magnon spectrum but also leads to spatially modulated magnetic ground states, i.e., a helix and a skyrmion lattice [14]. The Bragg scattering of magnons from these periodic magnetic textures results in a magnon band structure where the reciprocal lattice vectors of the associated Brillouin zone is determined by the DMI.

At the $\Gamma$ point of this Brillouin zone various resonances arise due to the backfolding of the spectrum, but only a few of them are magnetically active. In the helimagnetic phase, there are two magnetic resonances with finite spectral weight where the mean magnetization precesses clockwise or counterclockwise $[2,15]$. The skyrmion lattice phase is characterized by three magnetic resonances, including a breathing mode where the mean magnetization possesses an oscillating component along the applied magnetic field [16,17]. Quantitative agreement between theory and experiment was obtained for the microwave resonances observed in $\mathrm{MnSi}, \mathrm{Fe}_{1-x} \mathrm{Co}_{x} \mathrm{Si}$, and $\mathrm{Cu}_{2} \mathrm{OSeO}_{3}[18,19]$.

The low-energy magnon band structure of the helimagnetic phase for finite wave vectors $\mathbf{q}$ was studied in $\mathrm{MnSi}$ at zero field with inelastic neutron scattering by Janoschek et al. [20]. However, the obtained spectra were complex superpositions of magnon dispersions associated with the presence of multiple domains. The band structure was resolved by Kugler et al. [21] after preparing a single helimagnetic domain in $\mathrm{MnSi}$ using a small polarizing magnetic field, and the energies of 
the observed helimagnons were in quantitative agreement with theory. MnSi is especially well suited for a study of the helimagnon band structure because its characteristic energy scale is on the order of $0.1 \mathrm{meV}$, which can be resolved with state-of-the-art neutron spectrometers. For $\mathrm{Cu}_{2} \mathrm{OSeO}_{3}$, in contrast, the typical helimagnon bandwidths are an order of magnitude smaller, limiting inelastic neutron scattering studies to high-energy features of the magnon spectrum [22]. In the field-polarized phase, early studies of the magnon dispersion [23] of MnSi were not sensitive enough to observe the salient feature of nonreciprocity. It was, however, established later by Shirane et al. [6] and recently confirmed by Grigoriev et al. [24] and Sato et al. [7]. Moreover, the nonreciprocity of magnetic excitations was also demonstrated in the paramagnetic phase of MnSi using polarized neutrons by Roessli et al. [25].

In the present work, we study the evolution of the magnon spectrum in MnSi as a function of magnetic field covering the helimagnetic phase and the field-polarized phase. Our results illustrate the development of the magnetic structure factor across the continuous phase transition at the critical field $H_{c 2}$ separating the two phases, elucidating symmetry aspects of the magnon dispersion and its nonreciprocity. Remarkably, taking the resolution of the neutron spectrometers into account, the full neutron spectra are quantitatively explained by the theory for cubic chiral magnets using a single fitting parameter $\Gamma(H)$ for each field $H$ characterizing the intrinsic linewidth of the magnons.

In Sec. II we describe the instruments and the experimental conditions. In Sec. III we introduce the theoretical framework and provide an overview over the theoretically expected neutron spectra and their weights. In Sec. IV the experimental data are presented for various configurations and magnetic fields, and we conclude in Sec. V with a discussion.

\section{EXPERIMENTAL METHODS}

Our experiments were conducted using the cold-neutron triple-axis spectrometers MIRA [26,27] at the Maier-Leibnitz Zentrum in Garching, Germany, and TASP [28] at the PaulScherrer-Institut in Villigen, Switzerland, employing a cylindrical MnSi single crystal $(r=5 \mathrm{~mm}, h=30 \mathrm{~mm})$ oriented with the [001] direction along the cylinder axis. All inelastic scans were performed in the vicinity of the (110) Bragg reflection. The energies of the incident $\left(E_{i}\right)$ and scattered $\left(E_{f}\right)$ neutrons at MIRA and TASP were fixed at $E_{i}=$ $4.06 \mathrm{meV}$ and $3.5 \mathrm{meV} \leqslant E_{f} \leqslant 4.06 \mathrm{meV}$, respectively. At both instruments, a neutron guide defined the divergence of the neutrons impinging the monochromator. The collimation before and after the sample was 30 and 40 minutes for MIRA and TASP, respectively, yielding an energy resolution in the range $37 \mu \mathrm{eV} \leqslant \triangle E \leqslant 42 \mu \mathrm{eV}$. Higher-order neutrons were removed by a cooled Be filter.

Before each series of measurements at fixed magnetic field $\mathbf{H}$, the sample was heated above the critical temperature into the paramagnetic phase to remove any history-dependent effects. After cooling down the sample to the measurement temperature $T=20 \mathrm{~K}$, the magnetic field $\mathbf{H}$ was applied.

For the data analysis, the neutron spectra were deconvoluted using the open-source [29] software package TAKIN
[30-32], which employs the algorithm by Eckold and Sobolev [33] to calculate the four-dimensional instrumental resolution function. Using a four-dimensional deconvolution procedure, we gain quantitative information from our experimental data involving not only the magnon energies but also their spectral weights. This is especially important in the vicinity of the phase transition at $H_{c 2}$ where the helimagnetic bands would otherwise be indistinguishable due to their close proximity to each other in energy.

\section{THEORY OF THE NEUTRON SCATTERING CROSS SECTION IN CHIRAL MAGNETS}

\section{A. Low-energy theory of cubic chiral magnets}

The cubic chiral magnets at low energies are described by the free-energy density $[13,21] \mathcal{F}=\mathcal{F}_{0}+\mathcal{F}_{\text {dipolar }}+\mathcal{F}_{\text {corr }}$ that depends on the magnetization $\mathbf{M}=m \hat{n}$, with $m$ being the amplitude and $\hat{n}$ being the unit vector. The exchange contribution at low energies is given by [34]

$$
\mathcal{F}_{0}=\frac{\rho_{s}}{2}\left[\left(\nabla_{i} \hat{n}_{j}\right)^{2}-2 k_{h 0} \hat{n}(\nabla \times \hat{n})\right]-\mu_{0} m \hat{n} \mathbf{H},
$$

with $\rho_{s}$ being the exchange stiffness density and $\mathbf{H}$ being the applied field. The contribution $\mathcal{F}_{\text {dipolar }}$ is due to dipolar interactions, and $\mathcal{F}_{\text {corr }}$ is associated with higher-energy corrections. In Ref. [21] the latter term was chosen to be of the form $\mathcal{F}_{\text {corr }}=\frac{\rho_{s}}{2} \frac{\mathcal{A}}{k_{h}^{2}}\left(\nabla^{2} \hat{n}\right)^{2}$. At high fields $H>H_{c 2}$, the ground state is field polarized $\hat{n}=\mathbf{H} /|\mathbf{H}|$, and at lower fields $H<H_{c 2}$ a conical helix emerges.

For a field applied along the $z$ axis, the helix is given by $\hat{n}(z)=\left(\sin \theta \cos \left(k_{h} z\right),-\sin \theta \sin \left(k_{h} z\right), \cos \theta\right)$, and it is left handed for the sign of the Dzyaloshinskii-Moriya interaction $\left(k_{h 0}>0\right)$ chosen in Eq. (1). In zeroth order in the correction $\mathcal{A}$, the pitch vector $k_{h}$ is determined by $k_{h}=k_{h 0}$; a finite $\mathcal{A}$ slightly renormalizes the value of $k_{h}$. The cone angle $\cos \theta=H / H_{c 2}$ is determined by the magnetic field interpolating smoothly between the conical and field-polarized phases. The precessional dynamics of the magnetization is governed by the equation of motion $\partial_{t} \hat{n}=-\frac{g \mu_{B}}{\hbar} \hat{n} \times \mathbf{B}_{\text {eff }}$, with the effective field $\mathbf{B}_{\text {eff }}=$ $-\frac{1}{m} \frac{\delta F}{\delta \hat{n}}$, where $F=\int d \mathbf{r} \mathcal{F}$. The stiffness of the magnon dispersion is given by $\mathcal{D}=g \mu_{B} \rho_{s} / m=g \mu_{B} \mu_{0} H_{c 2}^{\text {int }} / k_{h}^{2}$, which can be expressed in terms of the internal critical field $H_{c 2}^{\text {int }}=$ $H_{c 2}-\mathrm{Nm}$ with the appropriate demagnetization factor $N$. Our experiments were performed at a temperature $T=20 \mathrm{~K}$ on MnSi where the pitch vector $k_{h}=0.036 \AA^{-1}$, the internal critical field $\mu_{0} H_{c 2}^{\text {int }}=0.53 \mathrm{~T}$, the susceptibility $\chi_{\mathrm{con}}^{\text {int }}=m / H_{c 2}^{\text {int }}=$ 0.34 , and the stiffness $\mathcal{D}=47.8 \mathrm{meV}^{2}$, with the $g$ factor $g \approx 2$ for $\mathrm{MnSi}$ [20,21]. Moreover, the high-energy correction was determined in Ref. [21] to be $\mathcal{A}=-0.0073$ [35]. This parameter set completely fixes the magnon dispersion, providing a parameter-free prediction as a function of applied field $\mathbf{H}$.

The differential cross section for inelastic neutron scattering is given by

$$
\frac{d^{2} \sigma}{d \varepsilon d \Omega} \propto\left[1+n_{B}(\varepsilon)\right] \operatorname{tr}\left\{\left(\mathbb{1}-\hat{G} \hat{G}^{T}\right) \chi^{\prime \prime}(\varepsilon, \mathbf{q})\right\},
$$

where $\mathbf{q}$ is the wave-vector transfer with respect to a nuclear reciprocal lattice vector $\mathbf{G}$, with $|\mathbf{q}| \ll|\mathbf{G}|$. The Bose factor $n_{B}$ ensures that the absorption of magnons with energy $\varepsilon$ 


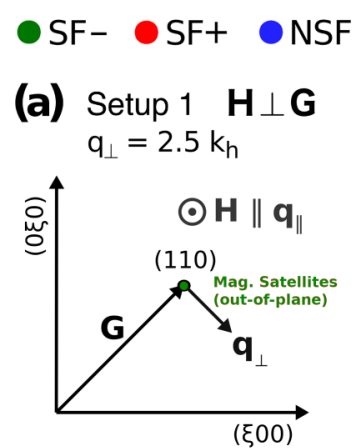
(b) Setup $2 \mathbf{H} \| \mathbf{G}$ $\mathrm{q}_{\perp}=2.5 \mathrm{kh}$
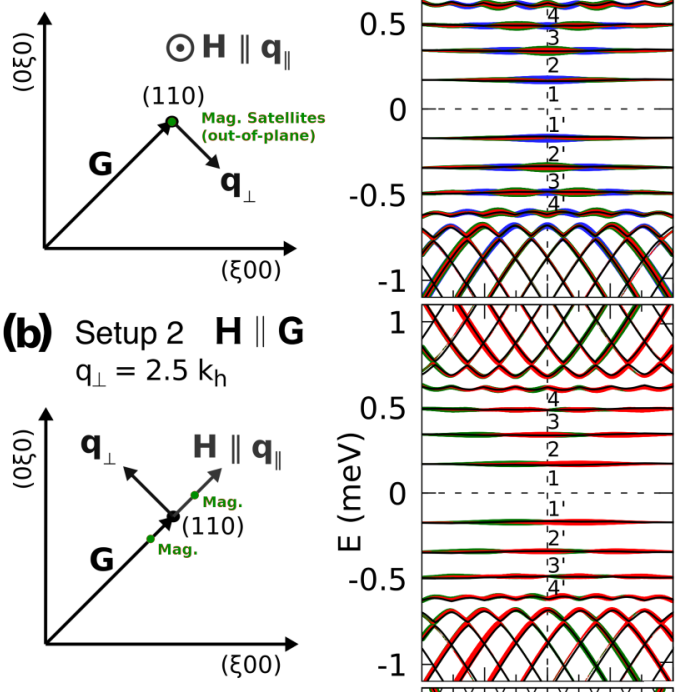
helical

$\mu_{0} \mathrm{H}_{\text {int }}=0.48 \mathrm{~T}$ conical

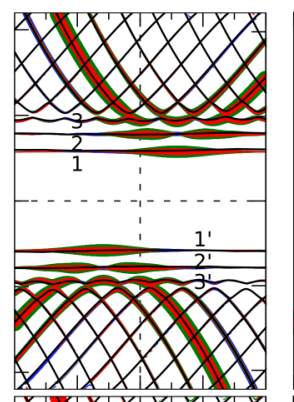

$\mu_{0} \mathrm{H}_{\text {int }}=0.61 \mathrm{~T}$

field-polarized

\section{(c) Setup $3 \mathbf{H} \perp \mathbf{G}$ $\mathrm{q}_{\perp}=0 \mathrm{kh}$}
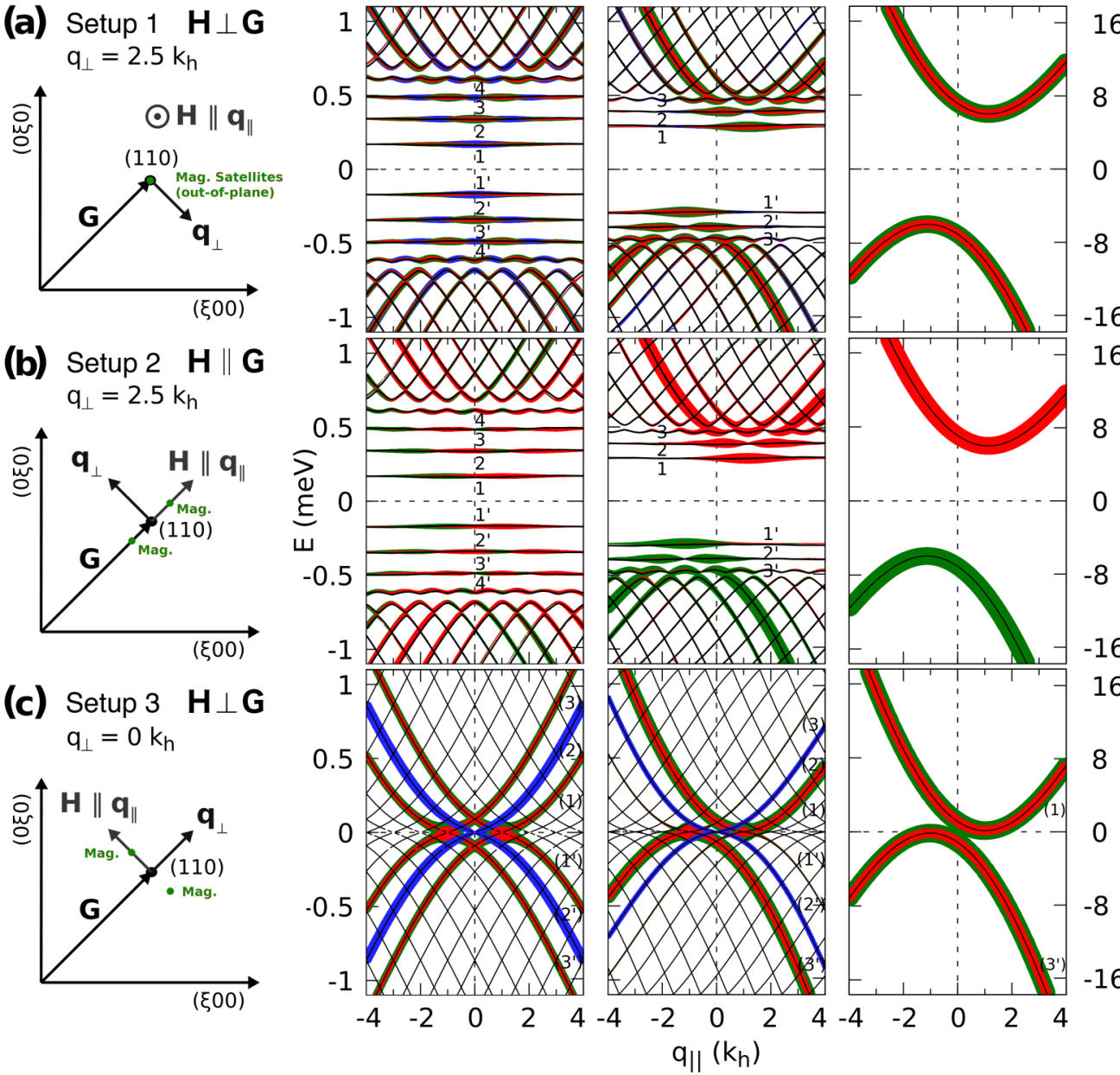

16

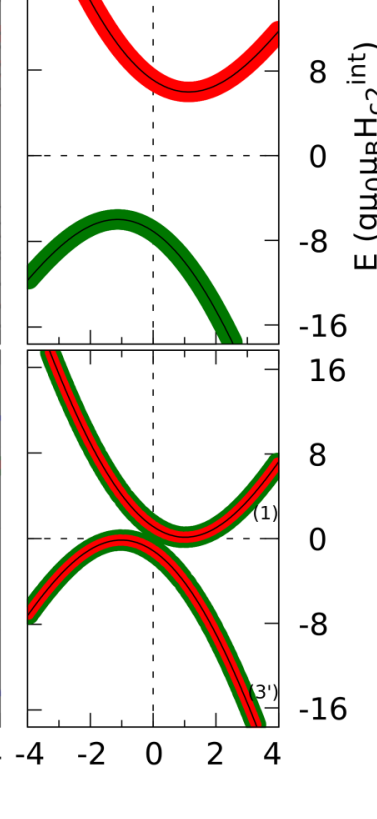

FIG. 1. Evolution of the magnon spectrum and spectral weights in a magnetic field $\mathbf{H}$. The three spectra for each setup represent the helical phase at zero field, the conical phase at intermediate fields, and the field-polarized phase at $\mu_{0} H_{\text {int }}>\mu_{0} H_{c 2}^{\text {int }}=0.53 \mathrm{~T}$. The magnon wave vector $q_{\|}$parallel to the field is shown on the horizontal axis, while $\mathbf{q}_{\perp} \perp \mathbf{H}$ is fixed. (a) and (b) show the spectrum for a fixed $\left|\mathbf{q}_{\perp}\right|=2.5 k_{h}$ and for an applied field perpendicular and along the nuclear reciprocal lattice vector $\mathbf{G}$, respectively. (c) shows the spectrum for a fixed $\mathbf{q}_{\perp}=0$ and $\mathbf{H} \perp \mathbf{G}$. The numbers indicate the observed peaks in Figs. 2-4. The theoretically expected spectral weights are represented by the shaded red and green lines corresponding to spin-flip ( $\mathrm{SF} \pm$ ) scattering and blue lines corresponding to non-spin-flip (NSF) scattering processes. The thickness of the shading scales linearly with the spectral weight except in in close proximity to the magnetic satellites $q_{\|}= \pm k_{h}$, where the spectral weight diverges and the shading instead is scaled logarithmically with the weight. Note that there is a finite magnon gap in the field-polarized phase that is, however, still small for the chosen field value $\mu_{0} H_{\text {int }}=0.61 \mathrm{~T}$.

is suppressed at low temperatures. The projection operator $P_{\hat{G}}=\mathbb{1}-\hat{G} \hat{G}^{T}$ arises from dipolar interactions between the neutron spin and the magnetization projecting onto the space perpendicular to the unit vector $\hat{G}=\mathbf{G} /|\mathbf{G}|$. All our experiments were performed with respect to $\hat{G}=(1,1,0) / \sqrt{2}$. We obtain the imaginary part of the susceptibility matrix $\chi_{i j}^{\prime \prime}$ of the magnetization with the help of linear spin-wave theory [21,36,37]. In principle, the trace in Eq. (2) can be decomposed into a sum of three contributions consisting of two spin-flip processes and one non-spin-flip process. For a neutron spin polarized along the magnetic field axis $\hat{H}=\mathbf{H} /|\mathbf{H}|$ the component $\hat{H}^{T} P_{\hat{G}} \chi^{\prime \prime} P_{\hat{G}} \hat{H}$ describes the non-spin-flip scattering event and does not contribute to $\mathbf{H} \| \mathbf{G}$ as $P_{\hat{G}} \hat{H}=0$. In the following discussion, it is instructive to distinguish these processes, although in our unpolarized scattering experiment all of them are added up according to Eq. (2).

\section{B. Magnon spectrum and spectral weights}

The theoretically expected magnon spectrum and the associated spectral weights are illustrated in Fig. 1 for the three experimental setups used. In setups 1 and 3 the magnetic field, $\mathbf{H} \perp \mathbf{G}$, is applied perpendicular to the nuclear reciprocal lattice vector, and $\mathbf{H} \| \mathbf{G}$ is used in setup 2. The dispersion is shown as a function of the magnon wave vector $q_{\|}$parallel to the applied field for a fixed wave vector $\mathbf{q}_{\perp}$ perpendicular to $\mathbf{H}$, that is, $\left|\mathbf{q}_{\perp}\right|=2.5 k_{h}$ for setups 1 and 2 and $\mathbf{q}_{\perp}=0$ for setup 3 . The temperature-independent spectral weights from the trace in Eq. (2) associated with two spin-flip scattering processes and a single non-spin-flip process are represented by the red, green, and blue shading, respectively. Whereas the spectra of setups 1 and 2 are the same, the weight distributions differ because the non-spin-flip scattering does not contribute in setup 2 as $\mathbf{H} \| \mathbf{G}$. The energy transfer is shown on the vertical axis, where 
positive and negative energies correspond to the creation and absorption of a magnon by the neutron, respectively.

Three representative spectra are shown in Fig. 1 for each setup: the helical phase at zero field, the conical phase at finite field $H_{\text {int }}<H_{c 2}^{\text {int }}$, and the field-polarized phase for $H_{\text {int }}>H_{c 2}^{\text {int }}$. Below the critical field $H_{c 2}^{\text {int }}$, the periodic magnetic texture leads to a band structure for the magnons. However, due to the screw symmetry of the helix magnon band gaps appear only for a finite perpendicular wave vector $\mathbf{q}_{\perp}$ as otherwise the Bragg scattering off the periodic magnetization associated with helical ordering is inactive [13]. For $\left|\mathbf{q}_{\perp}\right|=2.5 k_{h}$ the lowest three bands are basically flat at zero field, and in addition, the weights of the three channels are distributed over various helimagnon bands.

As the magnetic field increases, the weight of the non-spinflip scattering process in setup 1 decreases and vanishes at the critical field $H_{c 2}^{\text {int }}$. In the field-polarized phase only a single magnon branch remains, and it is sensitive only to spin-flip scattering in our setups. This branch corresponds to a parabola at low energies that is shifted by the DMI, giving rise to a nonreciprocal spectrum. Whereas in setup 1 both spin-flip scattering channels contribute equally, in setup 2 the weight of this branch is dominated by a single spin-flip process. For vanishing $\mathbf{q}_{\perp}=0$ in setup 3 the band gaps are absent at zero field, and the scattering weight is limited to three distinct helimagnon branches, which are centered around the nuclear Bragg peak $\left(q_{\|}=0\right)$ and the two magnetic satellite peaks $\left(q_{\|}= \pm k_{h}\right)$ of the static structure factor.

With increasing field the weight of the non-spin-flip contribution again decreases and vanishes in the field-polarized phase where only a single branch survives. Similar to setup 1 , both spin-flip processes contribute with equal weight to this branch at $H_{\text {int }}>H_{c 2}^{\text {int }}$. Moreover, the magnon excitations acquire a finite gap at $q_{\|}= \pm k_{h}$ when the field exceeds $H_{c 2}^{\text {int }}$.

The distribution of spectral weight reflects the symmetry of the dynamic susceptibility $\chi_{i j}^{\prime \prime}(\varepsilon, \mathbf{q})=-\chi_{j i}^{\prime \prime}(-\varepsilon,-\mathbf{q})$. Accordingly, the emission of a magnon with energy $\varepsilon(\mathbf{q})$, for example, in one spin-flip channel (red shading) possesses the same weight as the absorption of a magnon with energy $\varepsilon(-\mathbf{q})$ in the other spin-flip channel (green shading). In the fieldpolarized phase $H>H_{c 2}$ the spectrum exhibits a pronounced nonreciprocity, $\varepsilon\left(\mathbf{q}_{\perp}, q_{\|}\right) \neq \varepsilon\left(\mathbf{q}_{\perp},-q_{\|}\right)$, but only with respect to the wave vector $q_{\|}$longitudinal to the field. Neglecting magnetocrystalline anisotropies, there exists a combined rotation symmetry in spin and real space around the magnetic field axis, ensuring that the magnon energy $\varepsilon\left(\left|\mathbf{q}_{\perp}\right|, q_{\|}\right)$depends only on the amplitude and not on the direction of $\mathbf{q}_{\perp}$.

In the helimagnetically ordered phase, this rotation symmetry is broken by the magnetic ground state. However, the helix still possesses the screw symmetry, so that the magnon energy $\varepsilon\left(\left|\mathbf{q}_{\perp}\right|, q_{\|}\right)$still depends only on the amplitude of $\mathbf{q}_{\perp}$ even for $H_{\text {int }}<H_{c 2}^{\text {int }}$. The nonreciprocity $\varepsilon\left(\left|\mathbf{q}_{\perp}\right|, q_{\|}\right) \neq \varepsilon\left(\left|\mathbf{q}_{\perp}\right|,-q_{\|}\right)$ is still present at finite field and materializes, for example, in Fig. 1(c) as a shift of the band crossings in the conical phase away from $q_{\|}=0$. This shift can be attributed to the higher-energy correction $\mathcal{F}_{\text {corr }}$ and thus becomes less important at low frequencies.

Finally, at zero magnetic field the helix $\hat{n}(z)=\left(\cos \left(k_{h} z\right),-\right.$ $\left.\sin \left(k_{h} z\right), 0\right)$ is also invariant with respect to a $\pi$ rotation of spin and real space around the $x$ axis, which ensures that $\varepsilon\left(\left|\mathbf{q}_{\perp}\right|, q_{\|}\right)=\varepsilon\left(\left|\mathbf{q}_{\perp}\right|,-q_{\|}\right)$, so that the spectrum becomes reciprocal at $\mathbf{H}=0$. Whereas the spectrum is reciprocal at zero field, the weight distribution might remain nonreciprocal. For example, in a polarized neutron scattering experiment with a polarization longitudinal to $\mathbf{G}$ the weight distribution for spin-flip scattering processes will be asymmetric in $q_{\|}$even for $\mathbf{H}=0$ (see setup 2).

\section{EXPERIMENTAL RESULTS}

\section{A. Helimagnon bands at finite wave vector $q_{\perp} \perp \mathbf{H}$}

In order to probe the magnon spectrum at a finite wave vector $\mathbf{q}_{\perp}$ perpendicular to the applied field, experiments at the instruments MIRA and TASP (see Sec. II) were performed with a magnetic field along the crystallographic [001] and [110] directions, corresponding to setup 1 with $\mathbf{H} \perp \mathbf{G}$ and setup 2 with $\mathbf{H} \| \mathbf{G}$, respectively. A finite field was applied in order to prepare a state with only a single magnetic domain. Measurements were taken at different values of $\mathbf{q}_{\perp}$ [see Figs. 2(a) and 3(a)]. Here, we concentrate on the results with the reduced momentum transfer $\left|\mathbf{q}_{\perp}\right|=2.5 k_{h}$ and refer to the Supplemental Material [38] for the other values. The longitudinal momentum was chosen to be zero, so that the obtained neutron data correspond to cuts through the spectra of Figs. 1(a) and 1(b) at $q_{\|}=0$.

The evolution of the spectra with field as measured in setup 1 is shown in Fig. 2. Figure 2(b) shows the theoretically expected weight as a function of field, and Fig. 2(c) displays the neutron spectra. The data were collected at negative energies via absorption of magnons. The green lines were obtained by a convolution of the theoretical spectrum with the resolution function of the instrument. The blue lines indicate the contributions of individual helimagnon bands whose positions are also indicated by arrows. In addition, an intrinsic linewidth $\Gamma$ has been introduced which is approximated to be the same for all bands. The linewidth $\Gamma$ is the single fitting parameter as all other parameters were taken from previous measurements. At low magnetic fields four helimagnon bands are resolved. With increasing field, these bands come closer in energy and start to merge as the critical field $H_{c 2}$ is approached. Above the critical field, a single magnon excitation survives in the field-polarized state.

Figure 3 shows the corresponding spectra for setup 2 which were collected at positive-energy transfer through emission of magnons. Due to the parallel alignment $\mathbf{H} \| \mathbf{G}$ the non-spin-flip scattering does not contribute. As a result, the weight of the third band at low fields is suppressed in comparison to setup 1. The third band gains importance as the field increases and substantially contributes to the total weight close to the critical field. Above $H_{c 2}$, again, only a single magnon mode is detected.

\section{B. Nonreciprocal helimagnon dispersion for $q_{\|} \| \mathbf{H}$}

The magnon spectrum for vanishing $\mathbf{q}_{\perp}$ but finite wave vector $q_{\|}$along the field was experimentally probed with setup 3 at the TASP spectrometer with $\mathbf{H}$ applied along [110] so that $\mathbf{H} \perp \mathbf{G}$. This configuration corresponds to cuts through the spectra of Fig. 1(c) at the respective values of $q_{\|}$. As shown in Fig. 4(a), different values for $q_{\|}$were investigated [38] and distinguished from other spectra of nonmagnetic origin 

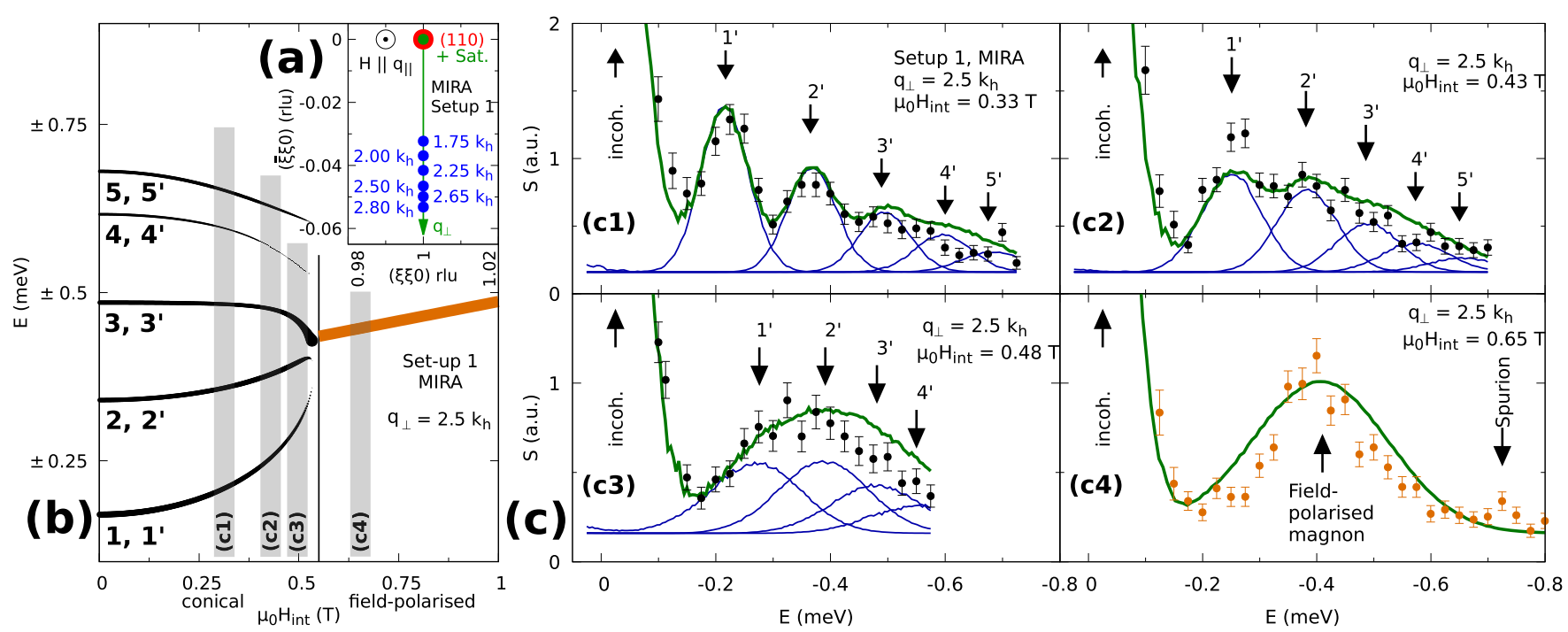

FIG. 2. Helimagnon bands at finite $\mathbf{q}_{\perp}$ with $\mathbf{H} \perp$ G. (a) Data were collected at different transferred momenta $\mathbf{q}_{\perp}$ (blue dots) perpendicular to the applied magnetic field. The red dot shows the position of the nuclear Bragg reflection $\hat{G}=\frac{1}{\sqrt{2}}(110)$ with $\mathbf{H} \perp \mathbf{G}$. The green dot depicts the projections of the out-of-plane helimagnetic satellite peaks. (b) Field dependence of the theoretically expected total weight at $\left|\mathbf{q}_{\perp}\right|=2.5 k_{h}$ corresponding to a cut through the spectra of Fig. 1(a) at $q_{\|}=0$. For unpolarized neutrons the weight at $q_{\|}=0$ is the same for positiveand negative-energy transfer. (c) The experimental data (dots) for different magnetic fields and a comparison to theory (green lines) after convolution with the experimental resolution. The blue lines and arrows indicate the position of the individual helimagnon bands taking into account a finite linewidth $\Gamma$. The peak numbers indicate the numbering of the dispersion branches in Fig. 1(a). A spurion was identified at $\mu_{0} H_{\text {int }}=650 \mathrm{mT}[38]$.

[39]. Here, however, we concentrate on only the magnons at $q_{\|}= \pm 2.5 k_{h}$.

The measured neutron spectra are shown in Figs. 4(c) and 4(d) and are compared with the theoretically expected spectral weights, which are shown in Fig. 4(b). Three helimagnon branches are observed in Fig. 4(c) below the critical field, $H<H_{c 2}$. With increasing positive field, the peaks at positive-energy transfer associated with branches 2 and 3 lose weight, whereas branch 1 gains weight as the critical field is approached. Near $H_{c 2}$, branch 1 continuously develops into the magnon excitation of the field-polarized phase. The experimental spectrum possesses a strong asymmetry with respect to the sign of the energy transfer (see peaks 1 and $\left.1^{\prime}\right)$ that is intrinsic and cannot be accounted for by the Bose factor in Eq. (2). This is explicitly demonstrated by measurements with reversed transferred momentum $q_{\|}$n as shown in Figs. 4(c4) and 4(d4). Instead of peak 1 at positiveenergy transfer, a strong magnon peak $\left(1^{\prime}\right)$ appears at negative-energy transfer, confirming the nonreciprocity of the spectrum.
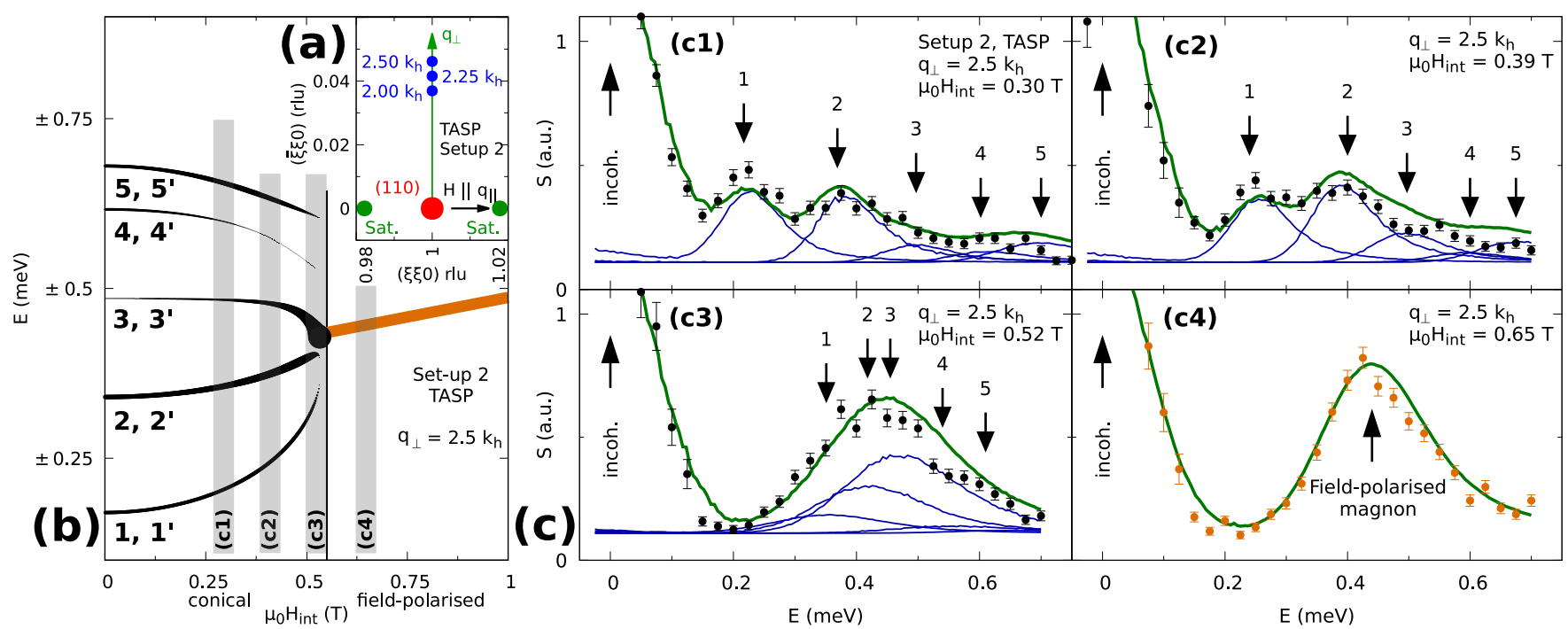

FIG. 3. Helimagnon bands at finite $\mathbf{q}_{\perp}$ with $\mathbf{H} \| \mathbf{G}$. The content of the panels is similar to Fig. 2, but here $\mathbf{H} \| \mathbf{G}$, leading to a different distribution of spectral weights. The green dots in (a) indicate positions of the magnetic satellite peaks due to helimagnetic order. The peak numbers indicate the numbering of the dispersion branches in Fig. 1(b). 

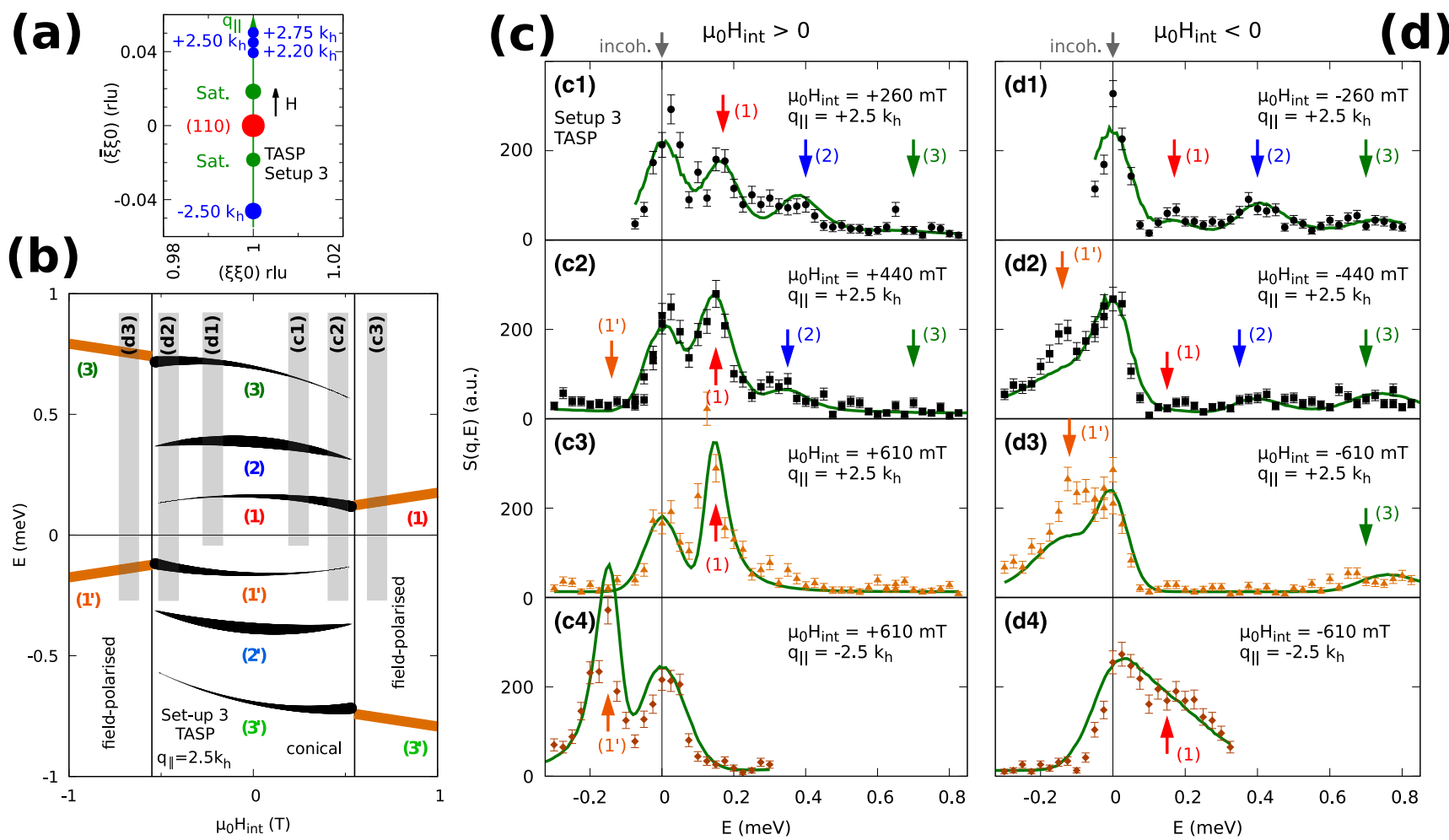

FIG. 4. Nonreciprocal helimagnon dispersion at finite $q_{\|}$. (a) Data were collected at different transferred momenta $q_{\|}$(blue dots) longitudinal to the applied magnetic field. The red dot shows the position of the nuclear Bragg reflection $\hat{G}=\frac{1}{\sqrt{2}}(110)$ with $\mathbf{H} \perp \mathbf{G}$. The green dots indicate the positions of the magnetic Bragg peaks of helimagnetic order. (b) Field dependence of the theoretically expected weight at $\mathbf{q}_{\perp}=0$ and $q_{\|}=2.5 k_{h}$ corresponding to cuts through the spectra of Fig. 1(c). For unpolarized neutrons, the weight at zero field is the same for positive- and negative-energy transfer, whereas nonreciprocity develops for finite $\mathbf{H}$. (c) The experimental data (dots) for different positive magnetic fields and a comparison with theory (green lines) after convolution with the experimental resolution. Three branches are detected in the helimagnetically ordered phase, whereas a single branch is present in the field-polarized phase for $\mu_{0} H_{\text {int }}>\mu_{0} H_{c 2}^{\text {int }}=530 \mathrm{mT}$ (arrows). (d) Experimental data for different negative magnetic fields. A comparison with (c) demonstrates the nonreciprocity of the spectrum under reversal of $\mathbf{H}$. The nonreciprocity with respect to $q_{\|}$is also explicitly demonstrated for $\mu_{0} H_{\text {int }}= \pm 610 \mathrm{mT}$ in (c4) and (d4). The peak numbers indicate the numbering of the dispersion branches in Fig. 1(c).

The nonreciprocity of the dynamic structure factor also becomes apparent after reversing the magnetic field. The reversal of $\mathbf{H}$ effectively inverts the magnon spectrum $\varepsilon\left(q_{\|}\right) \rightarrow \varepsilon\left(-q_{\|}\right)$ and also reshuffles the spectral weight. In Fig. 4(d) the neutron spectra are shown for negative fields that should be compared with the results of Fig. 4(c). Nonreciprocity is expected only at finite fields. However, for $\mu_{0} H_{\text {int }}=-260 \mathrm{mT}$ the spectrum is already clearly distinct from the one at positive field. Branch 2 has the largest spectral weight at $\mathbf{H}=0$. With increasing field its weight, however, decreases, and branch 3 gains in importance. It is this latter branch that now continuously connects with the magnon mode of the field-polarized phase. The degree of nonreciprocity of the neutron spectrum increases with increasing $|\mathbf{H}|$ and is particularly pronounced for $|\mathbf{H}|>$ $H_{c 2}$.

\section{DISCUSSION}

We investigated the magnon spectrum of MnSi for various values of the magnetic field covering the helimagnetically ordered and field-polarized phases using inelastic neutron scattering. For all investigated values of the magnetic field we found excellent quantitative agreement between the experimental neutron scattering data and theory after convolution with the instrumental resolution function [30-32]; the resulting quantitative theoretical spectra are shown as green lines in Figs. 2(c), 3(c), 4(c), and 4(d). Remarkably, both the dispersion $\varepsilon(\mathbf{q})$ of the magnon resonances and their spectral weights agree with parameter-free theoretical predictions. All parameters of the low-energy theory (see Sec. III) were determined by previous measurements.

After taking into account the full four-dimensional instrumental resolution function, the quantitative comparison between theory and experiment allowed us to extract also a field-dependent linewidth $\Gamma(H)$ (HWHM) of the resonances, which is the single fitting parameter necessary for the quantitative description of the experimental structure factor. A single value $\Gamma(H)$ was sufficient to describe the data of all magnon resonances for a given setup sufficiently well. Interestingly, for setup 3, i.e., for vanishing $\mathbf{q}_{\perp}=0$, the linewidth turned out to be negligible within the instrumental resolution. For setups 1 and 2, e.g., for $\left|\mathbf{q}_{\perp}\right|=2.5 k_{h}$, we obtained, however, finite values for $\Gamma$, hinting at a strong dependence of the magnon lifetime on the wave vector $\mathbf{q}_{\perp}$ perpendicular to the applied magnetic field.

The finite values for $\Gamma$ fitted to the data of setups 1 and 2 are summarized in Fig. 5. Values of approximately $\Gamma \sim 35 \mu \mathrm{eV}$ were found in the helimagnetically ordered phase, and larger 


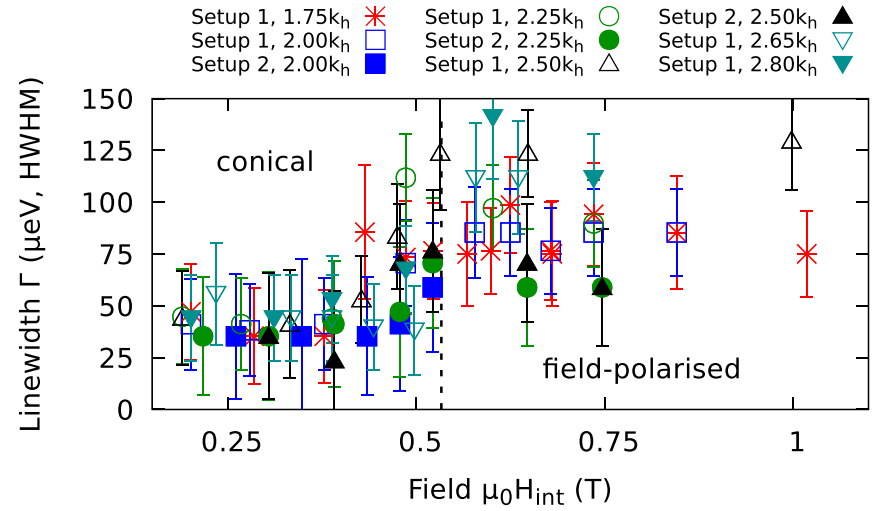

FIG. 5. Magnon linewidth $\Gamma$. The values of the half width at half maximum (HWHM) were obtained from fitting the spectra of setups 1 and 2 at different transferred momenta $\mathbf{q}_{\perp}$ and $q_{\|}=0$.

values $\Gamma \sim 85 \mu \mathrm{eV}$ were found in the field-polarized phase. For typical magnon energies of $\varepsilon \sim 0.2 \mathrm{meV}$ and $\varepsilon \sim 0.4 \mathrm{meV}$ in the helimagnetic and field-polarized phases, respectively, this corresponds to an effective damping parameter $\alpha=\Gamma / \varepsilon$ of approximately 0.17 and 0.21 . These values for $\alpha$ are roughly a factor of 3 larger than the findings of Schwarze et al. [18] using magnetic resonance measurements, i.e., for spin-wave excitations at $\mathbf{q}=0$. This is not unexpected as the phase space for magnon decay increases with increasing wave vector. Our findings suggest that the decay rate especially increases with the wave vector $\mathbf{q}_{\perp}$ pointing in a direction perpendicular to the magnetic field. The lifetime of the spin-wave modes probed in our experiments, in contrast to the uniform resonances, should be determined mostly by intrinsic effects like magnonmagnon interactions. We are not aware of any investigation of the magnon lifetime in chiral magnets, e.g., within the framework of nonlinear spin-wave theory, so our results for $\Gamma$ with its strong dependence on $\mathbf{q}_{\perp}$ should be clarified in future theoretical studies.

Nevertheless, such a detailed theoretical understanding of the experimentally observed magnon dispersion and spectral weights as presented in this work is unprecedented for chiral magnets in general and, in particular, for MnSi. Previous neutron scattering studies that considered the low-energy properties of chiral spin waves were often limited to the field-polarized phase and analyzed only the dispersion and not the spectral weight of the resonances $[6,7,23,24]$. Other works that investigated the spin waves in the helical and conical phases were not able to resolve the helimagnon band structure [6,20,22]. Kugler et al. [21] succeeded in resolving single helimagnon bands at small fields and also described their dispersion theoretically but not their spectral weights. Our results for $H \ll H_{c 2}$ are fully consistent with the data of Ref. [21].

In conclusion, using inelastic neutron scattering we have investigated the evolution of the band structure of the helimagnons in $\mathrm{MnSi}$ and the emergence of nonreciprocity as a function of the magnetic field $\mathbf{H}$ and the sign of the momentum transfer $\mathbf{q}$ covering the helical, conical helix, and field-polarized phases. The observed dispersion of the spin waves and their spectral weights are quantitatively explained by the low-energy theory of chiral magnets consisting of the symmetric exchange, Dzyaloshinskii-Moriya, Zeeman, and dipolar energies. These results set the stage for future explorations of the magnetization dynamics associated with the remaining phase in the phase diagram of chiral magnets housing the topological skyrmion crystal [40-42] as well as with the enigmatic non-Fermi-liquid regime in the paramagnetic phase of MnSi at high pressures [43].

\section{ACKNOWLEDGMENTS}

This work is based upon experiments performed at the MIRA instrument operated by FRM II at the Heinz MaierLeibnitz Zentrum (MLZ), Garching, Germany. Furthermore, this work is based on experiments performed at the Swiss spallation neutron source SINQ, Paul Scherrer Institute, Villigen, Switzerland. This work was part of the Ph.D. thesis of T.W. [32] and was supported by the DFG under Grant No. GE 971/5-1. M.G. is supported by the DFG via Grant No. SFB 1143 "Correlated Magnetism: From Frustration to Topology" and Grant No. GA 1072/5-1. A.B. and C.P. gratefully acknowledge financial support through DFG Grant No. TRR80 (project E1) and ERC Advanced Grant No. 291079 (TOPFIT). We thank R. Schwikowski for technical support and G. Brandl for his implementation of the helimagnon model which he created for Ref. [21] and which we further developed for this work.
[1] R. L. Melcher, Linear Contribution to Spatial Dispersion in the Spin-Wave Spectrum of Ferromagnets, Phys. Rev. Lett. 30, 125 (1973).

[2] M. Kataoka, Spin waves in systems with long period helical spin density waves due to the antisymmetric and symmetric exchange interactions, J. Phys. Soc. Jpn. 56, 3635 (1987).

[3] Y. Iguchi, S. Uemura, K. Ueno, and Y. Onose, Nonreciprocal magnon propagation in a noncentrosymmetric ferromagnet $\mathrm{LiFe}_{5} \mathrm{O}_{8}$, Phys. Rev. B 92, 184419 (2015).

[4] S. Seki, Y. Okamura, K. Kondou, K. Shibata, M. Kubota, R. Takagi, F. Kagawa, M. Kawasaki, G. Tatara, Y. Otani, and Y. Tokura, Magnetochiral nonreciprocity of volume spin wave propagation in chiral-lattice ferromagnets, Phys. Rev. B 93, 235131 (2016).
[5] R. Takagi, D. Morikawa, K. Karube, N. Kanazawa, K. Shibata, G. Tatara, Y. Tokunaga, T. Arima, Y. Taguchi, Y. Tokura, and S. Seki, Spin-wave spectroscopy of the DzyaloshinskiiMoriya interaction in room-temperature chiral magnets hosting skyrmions, Phys. Rev. B 95, 220406(R) (2017).

[6] G. Shirane, R. Cowley, C. Majkrzak, J. B. Sokoloff, B. Pagonis, C. H. Perry, and Y. Ishikawa, Spiral magnetic correlation in cubic MnSi, Phys. Rev. B 28, 6251 (1983).

[7] T. J. Sato, D. Okuyama, T. Hong, A. Kikkawa, Y. Taguchi, T.-H. Arima, and Y. Tokura, Magnon dispersion shift in the induced ferromagnetic phase of noncentrosymmetric MnSi, Phys. Rev. B 94, 144420 (2016).

[8] G. Gitgeatpong, Y. Zhao, P. Piyawongwatthana, Y. Qiu, L. W. Hariger, N. P. Butch, T. J. Sato, and K. Matan, Nonreciprocal 
Magnons and Symmetry-Breaking in the Noncentrosymmetric Antiferromagnet, Phys. Rev. Lett. 119, 047201 (2017).

[9] Y. Okamura, F. Kagawa, M. Mochizuki, M. Kubota, S. Seki, S. Ishiwata, M. Kawasaki, Y. Onose, and Y. Tokura, Microwave magnetoelectric effect via skyrmion resonance modes in a helimagnetic multiferroic, Nat. Commun. 4, 2391 (2013).

[10] M. Mochizuki, Microwave Magnetochiral Effect in $\mathrm{Cu}_{2} \mathrm{OSeO}_{3}$, Phys. Rev. Lett. 114, 197203 (2015).

[11] Y. Okamura, F. Kagawa, S. Seki, M. Kubota, M. Kawasaki, and Y. Tokura, Microwave Magnetochiral Dichroism in the ChiralLattice Magnet $\mathrm{Cu}_{2} \mathrm{OSeO}_{3}$, Phys. Rev. Lett. 114, 197202 (2015).

[12] M. Mochizuki and S. Seki, Dynamical magnetoelectric phenomena of multiferroic skyrmions, J. Phys.: Condens. Matter 27, 503001 (2015).

[13] M. Garst, J. Waizner, and D. Grundler, Collective spin excitations of helices and magnetic skyrmions: Review and perspectives of magnonics in non-centrosymmetric magnets, J. Phys. D 50, 293002 (2017).

[14] A. Bauer and C. Pfleiderer, Generic Aspects of Skyrmion Lattices in Chiral Magnets (Springer, Cham, 2016), pp. 1-28.

[15] M. Date, K. Okuda, and K. Kadowaki, Electron-spin resonance in the itinerant-electron helical magnet MnSi, J. Phys. Soc. Jpn. 42, 1555 (1977).

[16] M. Mochizuki, Spin-Wave Modes and Their Intense Excitation Effects in Skyrmion Crystals, Phys. Rev. Lett. 108, 017601 (2012).

[17] Y. Onose, Y. Okamura, S. Seki, S. Ishiwata, and Y. Tokura, Observation of Magnetic Excitations of Skyrmion Crystal in a Helimagnetic Insulator $\mathrm{Cu}_{2} \mathrm{OSeO}_{3}$, Phys. Rev. Lett. 109, 037603 (2012).

[18] T. Schwarze, J. Waizner, M. Garst, A. Bauer, I. Stasinopoulos, H. Berger, C. Pfleiderer, and D. Grundler, Universal helimagnon and skyrmion excitations in metallic, semiconducting and insulating chiral magnets, Nat. Mater. 14, 478 (2015).

[19] M. Weiler, A. Aqeel, M. Mostovoy, A. Leonov, S. Geprägs, R. Gross, H. Huebl, T. T. M. Palstra, and S. T. B. Goennenwein, Helimagnon Resonances in an Intrinsic Chiral Magnonic Crystal, Phys. Rev. Lett. 119, 237204 (2017).

[20] M. Janoschek, F. Bernlochner, S. Dunsiger, C. Pfleiderer, P. Böni, B. Roessli, P. Link, and A. Rosch, Helimagnon bands as universal excitations of chiral magnets, Phys. Rev. B 81, 214436 (2010).

[21] M. Kugler, G. Brandl, J. Waizner, M. Janoschek, R. Georgii, A. Bauer, K. Seemann, A. Rosch, C. Pfleiderer, P. Böni, and M. Garst, Band Structure of Helimagnons in MnSi Resolved by Inelastic Neutron Scattering, Phys. Rev. Lett. 115, 097203 (2015).

[22] P. Y. Portnichenko, J. Romhanyi, Y. A. Onykiienko, A. Henschel, M. Schmidt, A. S. Cameron, M. A. Surmach, J. A. Lim, J. T. Park, A. Schneidewind, D. L. Abernathy, H. Rosner, J. van den Brink, and D. S. Inosov, Magnon spectrum of the helimagnetic insulator $\mathrm{Cu}_{2} \mathrm{OSeO}_{3}$, Nat. Commun. 7, 10725 (2016).

[23] Y. Ishikawa, G. Shirane, J. A. Tarvin, and M. Kohgi, Magnetic excitations in the weak itinerant ferromagnet MnSi, Phys. Rev. B 16, 4956 (1977).

[24] S. V. Grigoriev, A. S. Sukhanov, E. V. Altynbaev, S.-A. Siegfried, A. Heinemann, P. Kizhe, and S. V. Maleyev, Spin waves in fullpolarized state of Dzyaloshinskii-Moriya helimagnets: Smallangle neutron scattering study, Phys. Rev. B 92, 220415(R) (2015).
[25] B. Roessli, P. Böni, W. E. Fischer, and Y. Endoh, Chiral Fluctuations in $\mathrm{MnSi}$ above the Curie Temperature, Phys. Rev. Lett. 88, 237204 (2002).

[26] R. Georgii and K. Seemann, MIRA: Dual wavelength band instrument, J. Large-Scale Res. Facil. 1, A3 (2015).

[27] R. Georgii, T. Weber, G. Brandl, M. Skoulatos, M. Janoschek, S. Mühlbauer, C. Pfleiderer, and P. Böni, The multi-purpose threeaxis spectrometer (TAS) MIRA at FRM II, Nuclear Instrum. Methods Phys. Res., Sect. A 881, 60 (2018).

[28] F. Semadeni, B. Roessli, and P. Böni, Three-axis spectroscopy with remanent benders, Phys. B (Amsterdam, Neth.) 297, 152 (2001).

[29] T. Weber, TAKIN software package, https://github.com/tweber/takin.

[30] T. Weber, R. Georgii, and P. Böni, Takin: An open-source software for experiment planning, visualisation, and data analysis, SoftwareX 5, 121 (2016).

[31] T. Weber, Update 1.5 to Takin: An open-source software for experiment planning, visualisation, and data analysis (PII: S2352711016300152), SoftwareX 6, 148 (2017).

[32] T. Weber, Dynamics at the orbital ordering phase transition in $\mathrm{MgV}_{2} \mathrm{O}_{4}$ and at the ferromagnetic phase transition in MnSi, Ph.D. thesis, Technische Universität München, 2016, http://nbn-resolving.de/urn/resolver.pl?urn:nbn:de:bvb:91-diss20170320-1339645-0-4

[33] G. Eckold and O. Sobolev, Analytical approach to the 4Dresolution function of three axes neutron spectrometers with focusing monochromators and analysers, Nucl. Instrum. Methods Phys. Res., Sect. A 752, 54 (2014).

[34] P. Bak and M. H. Jensen, Theory of helical magnetic structures and phase transitions in MnSi and FeGe, J. Phys. C 13, L881 (1980).

[35] Strictly speaking, the negative value of $\mathcal{A}$ requires further higherorder corrections in order to stabilize the theory.

[36] D. Belitz, T. R. Kirkpatrick, and A. Rosch, Theory of helimagnons in itinerant quantum systems, Phys. Rev. B 73, 054431 (2006).

[37] O. Petrova and O. Tchernyshyov, Spin waves in a skyrmion crystal, Phys. Rev. B 84, 214433 (2011).

[38] See Supplemental Material at http://link.aps.org/supplemental/ 10.1103/PhysRevB.97.224403 for additional measurements and information.

[39] D. Lamago, E. S. Clementyev, A. S. Ivanov, R. Heid, J.-M. Mignot, A. E. Petrova, and P. A. Alekseev, Lattice dynamics in the itinerant helical magnet MnSi, Phys. Rev. B 82, 144307 (2010).

[40] M. Janoschek, F. Jonietz, P. Link, C. Pfleiderer, and P. Böni, Helimagnons in the skyrmion lattice of MnSi, J. Phys.: Conf. Ser. 200, 032026 (2010).

[41] D. Fobes, T. Weber, J. Waizner, M. Kugler, A. Bauer, R. Georgii, P. Link, G. Ehlers, R. Bewley, C. Pfleiderer, P. Böni, M. Garst, and M. Janoschek, Spin excitations of the skyrmion lattice in MnSi, Bull. Am. Phys. Soc. (2018), http://meetings.aps.org/Meeting/MAR18/Session/B22.1.

[42] T. Weber, L. Beddrich, G. Tucker, M. Skoulatos, R. Georgii, A. Bauer, C. Pfleiderer, and P. Böni, Non-reciprocal magnons in the skyrmion phase of MnSi (unpublished).

[43] C. Pfleiderer, D. Reznik, L. Pintschovius, H. H. v. Löhneysen, M. Garst, and A. Rosch, Partial order in the nonFermi-liquid phase of MnSi, Nature (London) 427, 227 (2004). 